\title{
Comment tromper la censure. Correspondance 1915-1916 de Jules et Marie-Louise Puech
}

Wie man die Zensur umgehen konnte. Briefverkehr zwischen Jules und Marie-

Louise Puech (1915-1916)

Censorship and Correspondence 1915-1916 between Jules and Marie-Louise

Puech

\section{Rémy Cazals}

\section{OpenEdition}

Journals

Édition électronique

URL : http://journals.openedition.org/ceg/672

DOI : $10.4000 /$ ceg.672

ISSN : 2605-8359

\section{Éditeur}

Presses Universitaires de Provence

Édition imprimée

Date de publication : 18 novembre 2016

Pagination : 151-157

ISBN : 979-10-320-0087-8

ISSN : 0751-4239

\section{Référence électronique}

Rémy Cazals, «Comment tromper la censure. Correspondance 1915-1916 de Jules et Marie-Louise

Puech », Cahiers d'Études Germaniques [En ligne], 71 | 2016, mis en ligne le 18 mai 2018, consulté le 25 novembre 2020. URL : http://journals.openedition.org/ceg/672 ; DOI : https://doi.org/10.4000/ceg.672 


\title{
Comment tromper la censure
}

\author{
Correspondance 1915-1916 \\ de Jules et Marie-Louise Puech
}

\author{
Rémy CAZALS \\ Université Toulouse Jean-Jaurès
}

Ce texte bref n'est pas écrit par un germaniste; les personnages cités ne sont pas des Allemands, mais Jules Puech s'est trouvé à Verdun en 1915 et 1916 à quelques centaines, voire à quelques dizaines de mètres des Allemands qu'il ne désigne jamais du nom de "Boches ". S'il prend place dans la problématique "La lettre entre espace intime et sphère publique ", c'est que la correspondance entre mari et femme fait évidemment partie de l'espace intime, et que la sphère publique intervient ici par le moyen de la censure.

Jules Puech (1879-1957) et son épouse Marie-Louise Puech-Milhau (1876-1966) étaient tous deux issus de la bourgeoisie industrielle protestante du sud du Tarn. Ayant eu dans son enfance une gouvernante allemande, puis ayant rempli les mêmes fonctions dans une famille anglaise, avant d'enseigner pendant huit ans à l'université anglophone McGill au Canada, Marie-Louise était trilingue. Jules, docteur en droit en 1907, avait obtenu le poste de secrétaire général de la Société pour l'Arbitrage entre Nations, appointé par la Dotation Carnegie pour la Paix; il s'occupait également de la rédaction de la revue pacifiste La Paix par le Droit, une tâche à laquelle Marie-Louise participait, précieuse pour sa connaissance de l'anglais et de l'allemand lorsqu'il s'agissait de faire la revue des revues étrangères et de correspondre avec les pacifistes du monde entier, dont quelques-uns avaient obtenu ou allaient obtenir le prix Nobel de la Paix ${ }^{1}$.

Réformé pour raisons médicales, Jules Puech, qui avait 35 ans en 1914, voulut partir sur le front dans un régiment d'infanterie afın de participer à l'écrasement du militarisme allemand, principal obstacle, d'après lui, à l'établissement durable d'une Paix fondée sur le Droit ${ }^{2}$. Les deux époux furent séparés à partir de

1. Pour une biographie plus complète des Puech, voir Rémy Cazals, "L'intellectuel protestant était un couple: Jules et Marie-Louise Puech " dans le Bulletin de la Société de l'Histoire du Protestantisme français, 149, été 2003, "Parmi les intellectuels protestants, 1870-1940 ", p. 591610.

2. Rémy Cazals, "Faire la guerre pour établir la Paix par le Droit: Jules Puech (1915-1916) " dans le Bulletin de la Société de l'Histoire du Protestantisme français, 160, hiver 2014, "Les protestants français et la première guerre mondiale ", p. 399-416. 
mars 1915; Jules Puech connut le secteur calme de Verdun en 1915, puis les bombardements terribles à partir de février 1916, lors de la grande offensive Falkenhayn, et enfin la bataille de la Somme, à l'été, avant d'être évacué vers l'arrière pour une maladie cardiaque qui le rendait inapte au service armé. Restée à Paris, Marie-Louise prit sa succession dans les multiples activités liées aux organisations pacifistes, notamment la publication de la revue La Paix par le Droit.

Les Puech durent tenir compte d'une censure omniprésente pendant la guerre, ce qu'il faudra rappeler dans une première partie. On montrera ensuite comment on pouvait essayer de contourner cette censure, puis comment le soldat Puech utilisa divers codages pour faire passer à sa femme des informations interdites.

\section{Une censure omniprésente pendant la guerre}

Deux "mots de 14-18 " sont inséparables: censure et propagande, celle-ci qualifiée de " bourrage de crâne " par les combattants. Comme son nom l'indique, le bourrage de crâne consiste à faire entrer dans les esprits les affirmations officielles par tous les moyens d'information disponibles, par répétition et " matraquage ». La censure complète l'opération en essayant de faire disparaître toute idée subversive, toute idée qui viendrait contredire la vérité officielle. Le cinéma, le théâtre, la chanson populaire sont soumis à une censure tatillonne et parfois stupide ${ }^{3}$. Il en est de même de tout ce qui s'écrit. Les livres sont censurés, y compris les témoignages de combattants parus pendant la guerre. Il en est ainsi, par exemple, du livre de l'artilleur Lintier dont une page entière est supprimée parce qu'elle décrivait l'explosion d'un canon français de 75, provoquant la mort horrible de ses servants: le public ne devait pas apprendre que la fabrication de ces fameux canons pouvait être déficiente ${ }^{4}$. Devant le manuscrit de réflexion d'un homme des tranchées sur la paix future, écrit en 1915 par Albert Thierry, l'éditeur Ollendorf dut renoncer à le publier, la censure l'ayant complètement dénaturé: si elle laissa, en général, passer les remarques morales abstraites, elle supprima plus de $40 \%$ de la partie consacrée aux propositions concrètes parce qu'elles faisaient la part trop belle à l'Allemagne ${ }^{5}$. L'intégrale du livre de Thierry ne put être éditée qu'en décembre 1918.

La presse était particulièrement victime de la censure, avec des instructions strictes données aux journaux, et le contrôle des morasses avant parution. Tout le monde peut aller voir, dans les collections de périodiques de l'époque conservées dans les bibliothèques et les divers dépôts d'archives publiques, des numéros dont plusieurs lignes, voire des colonnes entières sont laissées en blanc. Je ne prendrai ici qu'un exemple, celui d'un quotidien du soir de Carcassonne

3. Voir Maurice Rajfus, La censure militaire et policière 1914-1918, Paris, Le cherche midi, 1999.

4. Paul Lintier, Le tube 1233, Souvenirs d'un chef de pièce (1915-1916), Paris, Plon, 1917, p. 30-31.

5. Voir Rémy Cazals, "Méditations sur la paix d'un combattant de 1915 ", dans Paroles de paix en temps de guerre, sous la dir. de Sylvie Caucanas, Rémy Cazals et Nicolas Offenstadt, Toulouse, Privat, 2006, p. 121-132. 
(Aude), La Dernière Heure, pas du tout subversif, annonçant au-dessus de son titre le slogan "L'Union de tous pour la Patrie ". Un des articles en une du journal du 16 octobre 1914, sur un peu plus d'une colonne, est entièrement censuré sauf le premier mot et la signature " Jacques Bonhomme ", évidemment un pseudonyme ${ }^{6}$. Si un passage litigieux était passé tout de même, le numéro du journal pouvait être saisi.

Sur les sujets particulièrement sensibles, comme la paix, le ministère prit d'abord des mesures ponctuelles, puis il en vint à interdire d'évoquer ce thème, même si on concluait en faveur d'une seule paix possible, par la victoire totale. Ainsi le préfet de l’Aude reçut-il, le 15 décembre 1916, un télégramme codé provenant du général commandant la $16^{\mathrm{e}}$ région militaire, dont voici la transcription: "Urgent. Ministre téléphone ce qui suit: consigne générale $n^{\circ} 152$ émanant de la présidence du conseil: "Interdire tous les articles pour ou contre la paix de façon à éviter des polémiques sur cette question ". Prière vouloir bien assurer en ce qui vous concerne exécution des instructions du ministre ${ }^{7}$. " On comprend qu'une revue qui contenait le mot " paix " dans son titre, comme $L a$ Paix par le Droit, eut particulièrement à souffrir de la censure.

Quant au courrier, celui qui venait de l'étranger était surveillé. Marie-Louise Puech ne cessait de demander à ses correspondants suisses, anglais ou américains de faire disparaître les mots " paix ", " pacifisme " et autres de même nature de l'en-tête de leurs enveloppes afın d'éviter les ennuis. Et on contrôlait aussi les lettres des militaires. Il était rigoureusement interdit à ceux-ci de dire à leur famille où ils se trouvaient sur le front: par de multiples recoupements, les espions allemands auraient pu reconstituer le déploiement de tous les régiments de l'armée française! Or savoir où se trouvait le fils, le mari ou le fiancé, cela paraissait une information indispensable. D'autre part, mieux valait ne pas critiquer les chefs, ne pas souhaiter la paix, ne pas décrire mutinerie ou fraternisation avec l'ennemi ${ }^{8}$. Bien sûr, il n'était matériellement pas possible pour le contrôle postal de lire la totalité des millions de lettres échangées tous les jours en franchise postale militaire; la censure procédait par sondages. Sur les 463 lettres envoyées par Jules Puech à sa femme entre mars 1915 et août 1916, une seule a été ouverte et refermée par un papier collant portant les mots "Contrôle postal militaire ". Cette lettre était anodine mais, le même jour, Jules Puech envoyait par la poste civile avec la complicité d'un permissionnaire, une lettre contenant une série de critiques à transmettre à des amis haut placés afin qu'ils en tiennent compte et qu'ils essaient de faire cesser les abus dénoncés.

6. Archives départementales de l'Aude, cote $2 \mathrm{R} 13$.

7. Ibid., cote $8 \mathrm{R} 26$.

8. Les rapports du contrôle postal sur la question des fraternisations sont utilisés dans le livre collectif Frères de tranchées, sous la dir. de Marc Ferro, Paris, Perrin, " coll. Tempus ", 2006. 


\title{
Contourner la censure
}

Jules Puech, sur le front, et Marie-Louise, à Paris, trouvèrent diverses astuces pour contourner la censure.

Pour Marie-Louise, il s'agissait de continuer à faire paraître la revue pacifiste. Celle-ci était imprimée à Nîmes et subissait une censure provinciale plus sévère que celle de Paris. Marie-Louise réussit à obtenir le changement d'autant plus profitable qu'on pouvait avoir des amis dans la place. Elle eut également l'habileté de s'abriter derrière Le Temps (quotidien officieux) ou L'Écho de Paris (du grand patriote Maurice Barrès) pour reprendre certaines nouvelles, mais en les développant à sa façon. Ce n'était pas toujours facile pour Marie-Louise mais son caractère combatif la rendait elle-même redoutable. Dans une lettre à son mari ( $1^{\text {er }}$ mars 1916), elle décrit ainsi un face à face avec " un vieil abruti " de la censure :

\begin{abstract}
Il m'a répondu qu'il y avait ordre de ne pas parler de la conférence de Zimmerwald parce que la minorité socialiste gagnait du terrain, qu'il fallait étouffer ce mouvement, etc., Mais ce n'est pas là le plus fort. Il est passé aux lettres de soldats où tout est tellement sabré qu'il n'en reste à peu près rien, peu importe que l'auteur soit agrégé, docteur ès-lettres, croix de guerre, médaille militaire, etc. En tête, ta lettre complètement biffée. «Monsieur, ai-je dit avec une indignation dans la voix, ceci est de mon mari qui se bat dans le secteur de V... en ce moment; ces lignes sont empreintes du patriotisme le plus sain; donnez-moi des explications car je ne puis comprendre pourquoi vous les supprimez!» Le monsieur a été " estabaousit " " et il a dit tout de suite: "Eh bien, puisque ceci est de Monsieur P..., je vais le laisser passer.» Et il a continué à tourner la page, où le crayon bleu continuait de remplir son rôle. Voici l'explication et elle vaut la peine qu'on la retienne: M. Lefèvre de Bremaigne, ancien diplomate, réactionnaire fieffé, croit que cette guerre, loin d'être la dernière, sera la "matrice» de guerres innombrables; de plus, l'histoire enseigne qu'il y a toujours eu des guerres, c'est une preuve qu'il y en aura toujours; donc il ne faut pas accréditer l'idée que cette guerre pourrait être la dernière et il ne faut pas laisser dire aux soldats qu'ils se battent pour éviter à leurs enfants d'en faire autant.
\end{abstract}

$\mathrm{Au}$ front, les militaires pouvaient recevoir sans problème les journaux conformistes. Mais La Guerre sociale, malgré l'évolution patriotique de son directeur, exhalait toujours un parfum subversif, si bien que Gustave Hervé finit par rebaptiser son journal LaVictoire! L'Humanité, que les Puech considéraient comme le quotidien le plus intéressant, devait être envoyé à Jules sur le front habilement caché dans Le Temps!

Pour s'exprimer en toute liberté, Jules Puech envoya quelques lettres par la poste civile qui était beaucoup moins surveillée. Sur le front, les militaires n'y avaient pas accès, mais ils pouvaient confier leur enveloppe, timbrée, à un permissionnaire qui la mettrait à la boîte en arrivant chez lui. Ainsi, la lettre écrite près de Verdun le 24 novembre 1915, porte-t-elle le cachet de la poste de Bollène (Vaucluse). Jules Puech eut encore recours à cette astuce pour une lettre du 26 décembre 1915 contenant une série de critiques et de propositions d'améliorations :

9. Occitanisme pour " très étonné ", " sidéré ". 
Voici ce que P. Barthe pense et dit à Gallieni : les débats de la Chambre ont mis en lumière des choses rigoureusement exactes. Il faut absolument qu'elles soient modifiées, sans quoi, comme l'a dit Hervé, on aura après la guerre une vague d'antimilitarisme forcené. Sur le chapitre des permissions : leur cours a été stupidement entravé ; si l'on s'efforce sincèrement de remédier, tout ira bien. Mais c'est essentiel: il faut, il faut absolument qu'on améliore la situation du soldat. Situation matérielle et situation morale seront améliorées par les mêmes mesures car tout se tient. il faut qu'en descendant des tranchées les hommes aient une période de vrai repos; on conçoit qu'il faille prendre de ces hommes-au-repos pour des travaux utiles, mais il ne faut pas que, sous prétexte d'obéir à un ordre de faire ces travaux, on fasse partir des hommes par des temps horribles pour ces travaux rendus précisément impossibles à cause du temps; il faut leur laisser le temps de laver, de se nettoyer.

On peut noter ici une précaution supplémentaire: Jules Puech se désigne luimême comme P. Barthe, pseudonyme qui était transparent pour son épouse ${ }^{10}$.

\section{Tromper la censure par des codages}

Mais le recours à la poste civile ne pouvait qu'être ponctuel. Pour tromper la censure éventuelle du courrier militaire, Jules Puech eut recours à des codages d'amateur, à distinguer des codes professionnels utilisés dans les armées et les administrations ${ }^{11}$. On peut en citer trois formes principales : les pseudonymes, les périphrases de connivence, les acrostiches.

Pour ne pas risquer d'ennuis, même si les lettres par la poste militaire avaient un contenu peu subversif, Jules Puech se désignait parfois par des pseudonymes, comme on l'a vu plus haut. P. Barthe revient souvent, et aussi Jules Helle qu'il avait utilisé avant la guerre dans des articles. Sa signature habituelle était Jules L. Puech (L pour Louis).

Les périphrases de connivence devaient évidemment être obscures pour la censure et facilement compréhensibles par Marie-Louise. On en trouve pour désigner de grands personnages égratignés: "le compatriote de Drevet " est le général Joffre, natif du département des Pyrénées-Orientales comme Drevet, ami des Puech; "le compatriote de Cim " désigne le président Poincaré, citoyen, comme l'ami Cim, de Bar-sur-Aube. D'autres périphrases indiquent une localisation géographique: "la ville du fils Weiss " (encore un ami), dont il est souvent question, est Verdun, et, dans le secteur de Verdun, "le gibet " désigne la colline de Montfaucon. Il faut comprendre le nom de village de Morvillars quand la lettre de Jules évoque un maréchal de Louis XIV décédé. Dire que des camarades sont allés chez Alexis François signifie que, le régiment étant cantonné près de la frontière, des soldats ont déserté en passant en Suisse (l'ami François vivait dans cet heureux pays neutre). Le 12 juin 1916, par la poste civile, Jules envoie une lettre qui annonce un télégramme codé afin d'organiser

10. Barthe était le nom du grand-père maternel de Jules, et l'entreprise industrielle familiale portait le double nom Puech-Barthe.

11. Des lettres de Jules Puech sont présentées dans l'exposition " Cacher, Coder, 4000 ans d'écritures secrètes " et son catalogue, Figeac, musée Champollion, 2015. 
une éventuelle rencontre dans une gare de la région parisienne où son régiment risque de passer:

Si nous passons par Noisy-le-Sec, je tâcherai de te télégraphier et tu t'ingénieras à deviner; par exemple " 15 kilos secs " pourraient vouloir dire que c'est le 15 que nous partons pour Noisy-le-Sec; " 16 volumes pour Paul " pourraient vouloir dire que le 16 on va au Bourget. Ces deux gares régulatrices, en effet, peuvent être choisies l'une ou l'autre. Si tu lisais: " 17 volumes Rodenbach pour Paul ", tu saurais que nous partons le 17 pour la Belgique en passant par Le Bourget. Je ne sais pas trop si ce sera toujours aussi facile de trouver des équivalents et je ferai pour le mieux, me fiant aussi à ta perspicacité. Cim indiquerait l'idée de Meuse ; évêché, l’idée de Verdun ; total, l'idée de Somme ; vigne, l’idée de Champagne, etc.

Au début de l'offensive de la Somme, lorsque son régiment avance vers l'est, le codage géographique devient presque un jeu. Pour désigner des villages, Jules Puech emploie les expressions: " Nous sommes passés par... le héros d’Elseneur, puis par la matière de la pantoufle de Cendrillon. "Et il y a encore " le chien de Vitalis " et " le village dont le nom évoque le gazon tondu ${ }^{12}$. " On frémit à l'idée que des espions allemands férus de culture française auraient facilement décrypté ces expressions et pu localiser le parcours du $365^{\mathrm{e}}$ régiment d'infanterie!

Enfin, Jules Puech pratique assez souvent l'acrostiche pour annoncer des nouvelles graves. Ainsi, le 11 février 1916, alors qu'on s'attend à une attaque qui se produira en effet, mais dix jours plus tard: "On évacue les villages." Ou encore: "Commandant $6^{e}$ bataillon suicidé hier matin secret demandé. " La pratique de l'acrostiche est un exercice acrobatique car les premières lettres de plusieurs lignes doivent servir à constituer le mot ou la phrase que l'on veut cacher. Pour annoncer qu'il se trouve cantonné au village de Montzéville, la difficulté vient du fait que peu de mots commencent par la lettre $z$. Alors, il écrit "ze ne te dis pas... " et il est obligé de commenter le passage par la remarque: "Quel style!"

Une dernière catégorie de codages nous ferait entrer dans le domaine de l'intime. J'en ai découvert certains, entrevu d'autres. Mais je ne les dévoilerai pas et je me contenterai d'évoquer ici les quelques fleurs, aujourd'hui séchées, qui restent encore accrochées à certaines lettres comme témoignage d'amour ${ }^{13}$.

À la place d'une conclusion, pas vraiment nécessaire après ce court papier, je placerai plutôt un épilogue en évoquant les Puech pendant la deuxième guerre mondiale, sous l'Occupation, une époque au cours de laquelle, selon Marie-Louise elle-même, mieux valait que la main gauche ignore ce que faisait la main droite. Elle s'occupait d'un réseau d'aide à des intellectuelles étrangères réfugiées en France ou bloquées par la guerre, Polonaises, Tchécoslovaques, juives du pays de Bade, etc. Recevant de l'argent venant de Suisse ou des États-Unis par les Quakers ou les Unitariens, elle était obligée d'organiser des rencontres clandestines avec un émissaire nommé Field, qui devenait Monsieur Champ dans les télégrammes. L'arrestation de l'amie polonaise Maria Wisti par la Gestapo fut traitée sous le

12. Hamelet, Vaire, Cappy, Herbécourt.

13. Sur l'ensemble de ces courriers, voir Jules et Marie-Louise Puech, Saleté de guerre! correspondance 1915-1916 présentée par Rémy Cazals, Paris, Ampelos, 2015. 
thème " la maladie de Maria me fait trop de peine ", et les tentatives de Jules Puech de faire intervenir des amis haut placés étaient décrites comme: "il écrit à un de nos amis dont les fonctions pourraient enrayer cette maladie ". Efforts qui pouvaient aboutir tant qu'on restait entre Français, mais avec la Gestapo ce n'était plus la même chose et il ne reçut " aucune réponse quant au traitement à suivre ". Deux réfugiées fuyant les nazis de Prague à Paris, puis de Paris vers le Tarn, mère et fille, juives, reçurent de l'argent et réussirent à passer en Suisse. On écrit à Marie-Louise, à leur propos: "Se trouvant subitement plus fatiguées, et pensant qu'un changement d'air leur était nécessaire, elles sont parties précipitamment pour essayer de rejoindre leur amie Marinette. " Ce qui signifie: se trouvant menacées, elles sont parties pour la Suisse (sachant que l'amie Marinette vivait à Genève). On trouve là un écho précis du passage chez Alexis François rencontré plus haut en 1916. Mais l'action des Puech pendant la deuxième guerre mondiale est une autre histoire, déjà exposée ailleurs ${ }^{14}$.

14. Rémy Cazals, Lettres de réfugiées, Le réseau de Borieblanque, Des étrangères dans la France de Vichy, Paris, Tallandier, 2003, et Jules Puech, Lettres au maréchal Pétain qui n'a pas eu la bonne idée de mourir plus tôt, Paris, La Découverte, 2016. 
